\title{
Determination and Enrichment of ATC by Optimally Localized TCSC via Hybrid Algorithm
}

\author{
Amjad Said \\ International Maritime College Oman \\ Suhar Port Falaj Al Qabail, Suhar OM ,Oman \\ amjadsaid231@gmail.com
}

\begin{abstract}
In the deregulated power system, the Available Transfer Capability (ATC) determination as well as enrichment seems to be a challenging issue. Thus, in this paper, the Thyristor-Controlled Series Compensation (TCSC) devices are incorporated to maximize the power transfer transactions. The TCSC is a series model of Flexible Ac Transmission System (FACTS) devices that are good in enhancing the ATC. Further, the optimal location for TCSC in and the number of compensations that need to be given by TCSC to the power system need to be determined. Hence, the Grasshopper Optimization with Genetic Algorithm (GOAGA) optimization model is introduced in this research work. Finally, the experimentation is done in MATLAB under two benchmark bus systems like IEEE 14 and IEEE 39.
\end{abstract}

Keywords: ATC Computation; FACTS; TCSC devices; ATC Enrichment; GOAGA model

\begin{tabular}{ll} 
Nomenclature & \\
\hline Abbreviations & Descriptions \\
\hline UPFC & Unified Power Flow Controller \\
ATC & Available Transfer Capability \\
TTC & Total Transfer Capability \\
TCSC & Thyristor-Controlled Series Compensation \\
TC & Transfer Capability \\
SVR & Support Vector Regression \\
STATCOM & Static Compensator \\
SAPSO & Self-Adapted Particle Swarm Optimisation \\
PSO & Particle Swarm Optimization \\
PS & Power System \\
MFO & Moth Flame Optimization \\
MA & Marginal Availability \\
GOA & Grasshopper Optimization Algorithm \\
GENCOs & GENeration COmpanies \\
GA & Genetic Algorithm \\
CPF & Continuation Power Flow \\
DISCOs & DIStribution COmpanies \\
DATC & Dynamic Available Transfer Capability \\
CSO & Cat Swarm Optimization \\
OPF & Optimal Power Flow \\
CBM & Capacity Benefit Margin \\
FACTS & Flexible Ac Transmission System \\
\hline
\end{tabular}

\section{Introduction}

In a compound electrical system, the power framework which is amazingly massive that incorporates power generation systems, transmission systems and conveyance organizes alongside loads, and these systems are being extended everywhere throughout the immense topographical zone. In the electric power generation, transmission, and distribution, the electric power demand is expanding and thus 
interminable endeavours are being carried out. These businesses continue to elevate their methods to support the structure of the serious electric power advertise in the deregulated condition [6] [7] [8] [9] [10] [11]. The rebuilding of the electric industry is more common these and their significant aim are to exchange electric power with lower prices, so as to advance serious electric markets. Electric manufacturers are deregulated all through the world with the target of producing forceful markets to purchase and sell electric power. A mass of new specialized difficulties are showcased by these deregulated power to supporters of the power advertise benefactors. In the region of transfer capability of transmission systems of a proficient organization, gigantic investigations are being made by the competitive market to satisfy the demands of the consumers. In a rebuilt situation, all GENCOs and DISCOs endeavor to offer for the most productive exchanges.

TC alludes to the capacity of the transmission system to move electric power reliably from a territory of flexibly to a region of interest by the method of all transmission lines (or ways) between two territories under a given working condition. With the guide of more straightforward power framework models like DC load flow estimate, the calculation of the Transfer capacities turns out to be very simpler. Further, while contrasted with the AC model, the DC model is increasingly ideal in specific conditions in which the beneficial information is inaccessible or unsure because of a long time period examination [12] [14] [15].

ATC in the physical transmission organize is the estimation of the rest of the exchange capacity that is deserted for playing out the helper commercial action well beyond the recently devoted employments [13]. All in all, an enormous amount of analysts was experienced to create ideal calculations and techniques for registering ATC. Among them, "linear incremental power flow" is one the anticipated answer for improving ATC and this model depended on the direct gradual power flow. Aside from this, it experiences downsides like the absence of the thought of the nonlinear impacts of responsive power and voltage. Nonetheless, the operational issues can be taken care of evenly with OPF strategy, yet here the exactness of ATC calculation is lower because of the usage of the approximations. In this way, it is fundamental to build up a well-suited strategy for improving ATC utilizing TCSC gadgets, since FACTS gadgets are anguishing from the genuine emergency during ideal area assurance. Moreover, the optimization algorithms [21] [22] [23] [24] [25] can be implied for the optimal placement of TCSC in the PS.

- The major contribution of this research work is depicted below:

- The TCSC, a series FACTS device is incorporated into the PS for enhancing the ATC.

- In the bus system, the optimal placement of TCSC and compensation level to be provided is decided by the GOAGA model.

- The introduced GOAGA model is the hybridized form of GA and GOA optimization approaches.

The rest of the paper is organized as Section 2 reviews the fascinating works undergone with respect to the current paper. Section 3 tells about computation and enrichment of ATC with TCSC device: an outline. Section 4 describes the introducing GOAGA model for ATC enhancement. The resultant acquired with the GOAGA model is discussed in Section 5. A strong conclusion is given to this research work in Section 6.

\section{Literature Review}

In 2010, Krishna et al. [1] have utilized TCSC devices with the target of enhancing ATC. In addition, they have utilized the SAPSO algorithm to achieve the goal by optimizing the system parameters of TCSC. In 2015, Srinivasan et al. [2] have developed "DATC with SVR" in the restructuring PS environment. Further, concerning the boundary of the potential energy surface, they have computed the DATC. This computed DATC was fed as input to SVR for DATC determination. In 2015, Mohseni et al. [3] have considered "SSSC, STACOM and UPFC" of the FACTS in order to reveal the ATC enrichment. In addition, they have developed "a novel current based modeling and optimal location strategy" of these controllers. In 2016, Nireekshana et al. [4] have investigated the necessity of incorporating FACTS into the deregulated operation of PSs in order to enhance the ATC. Here, the power transfer transactions were upgraded with FACTS devices. The voltage profile as well as the thermal limits was considered with the CPF method in ATC. SVC and TCSC's controlling parameters were localized using the CSO. In 2016, Pasam and Manohar [5] have introduced two heuristic approaches with the target of ATC assessment in the PS. In the initial contribution, the "continuation repeated power flow, repeated optimal power flow, radial basis function neural network, Backpropagation Neural Network, and adaptive neuron fuzzy inference system" were merged to compute ATC. Then, in the subsequent contribution, a vast count of ATC values was acquired. Among them, the specific ATC was selected with respect to "social, economic, deregulated environmental constraints". 


\section{Computation and Enrichment of ATC with TCSC Device: An Outline}

\subsection{Necessity of Connecting ATC with TCSC Devices}

In general, ATC is said to be "a measure of the transfer capability remaining in the physical transmission network for future commercial activity over and above already committed uses". The transfer of power takes place from GENCOS to DISCOS and it is determined by ATC. In the case of the power transfer along the transmission lines, the "reactive and real power" along MA is being a crucial factor. In some of the transmission lines, the upper bound of the ATC is set in prior, which is unsatisfactory to the consumer's demands. Therefore, ATC needs to be enhanced and in this research work, a series of FACTS device known as TCSC is deployed. "A typical TCSC has a series capacitor $\mathrm{C}_{\mathrm{s}}$ in parallel with a thyristor-controlled reactor $\mathrm{L}_{\mathrm{s}}$ ". Fig. 1 and Fig. 2 show the circuit diagram of TCSC and its equivalent model, respectively. Further, in TCSC, the variable series compensation is utilized to

- Improve the loadability as well as the base-power flow of the series compensated line.

- Additional losses in the compensated line from the enhanced power flow; and

- Increased responsiveness of power flow in the series compensated line from the outage of other lines in the system.

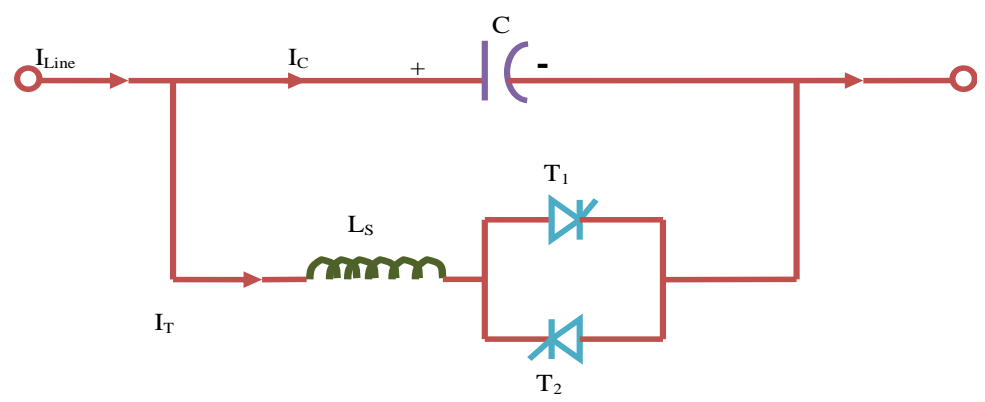

Fig. 1. Functional diagram of TCSC

The equivalent impedance $\mathrm{Z}_{\mathrm{eq}}$ is computed mathematically from the variable inductor $\mathrm{L}$ that is connected across the capacitor $\mathrm{C}$. This is shown in Eq. (1).

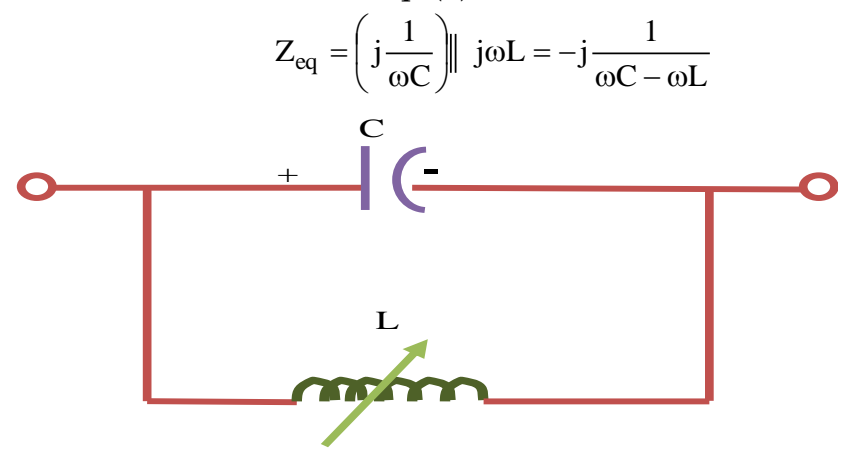

Fig. 2. Equivalent circuit of TCSC

\subsection{ATC Computation}

"Mathematically, ATC is defined as the Total Transfer Capability (TTC) less the Transmission Reliability Margin (TRM), less the sum of existing transmission commitments and the capacity benefit margin (CBM)". This is expressed in Eq. (2) and this formula is based on a commitment less transmission line.

$$
\mathrm{ATC}=\mathrm{TTC}-\mathrm{TRM}-\mathrm{CBM}
$$

TRM: it is defined as, "the amount of transmission transfer capability necessary to ensure that the interconnected transmission network is secure under a reasonable range of uncertainties in system conditions". CBM: it is defined as, "amount of transfer capability reserved by load-serving entities to ensure access to generation from interconnected systems to meet generation reliability requirements". TTC: it is defined as, "The amount of electric power that can be transferred over the interconnected transmission network reliably while meeting all the set of defined Pre- and Post- contingency system 
conditions". Further, the values of TTC, CBM, and TRM need to be computed for evaluating ATC. All these computational steps are discussed in the upcoming sections:

\section{3 TTC, TRM and CBM Assessment for ATC Computation: A General Procedure}

The steps involved in this computation are discussed below:

Step 1-Select the type of case, whether contingent or normal from the contingency list

Step 2-The stimulation of the load level takes place with the aid of the normal distribution.

Step 3-If there lacks power transfer $(\lambda=0)$, then establish the base case power flow and solve it. In the absence of violations move to step 4 or else set the load level as zero and move back to step 2 .

Step 4-Use RPF for even a step increase in the transfer level of the power (i.e. $\lambda$ augmented by $\Delta \lambda$ ).

Step 5-Then, establish the power flow problem and solve it. The acquired resultant is compared with the results from step 3. In the presence of violation, the transfer power can be rejected and its transfer rate is reduced to the utmost minimum value. Then, proceed to step 6 . Until the violation of the limit, the value of $\lambda$ is decreased by $10 \%$ of $\Delta \lambda$

Step 6-Subsequently, for the chosen case, the TTC is computed at the load level with maximal $\Delta \lambda$ value. Until the achievement of the TTC convergence, the load is kept on stimulating by moving back to step 2. Further, proceed to step 7.

Step 7-Then, select the other cases, one by one, and compute TTC as per the above procedure. Then, move to Step 8.

The computed TTC is lower for the "source/sink transfer case". On the other hand, during the computation of TRM, ignore step 2 and after TTC computation is done using Step 3 or Step 5, proceed directly to Step 6.

CBM Allocation: After the allocation of CBM into the ATC, the ATC is computed with Eq. (2), "If TTC isn't sufficient to supply CBM, then ATC will be negative" or CBM is defined as the firm transfers while computing TTC. With all these parameters, the ATC can be computed. But, while connecting TCSC to ATC, there is a necessity to decide its localization as well as compensation level that needs to be given to TCSC buses.

\section{Introducing GOAGA Model for ATC Enhancement}

\subsection{Objective Function and Solution Encoding}

The prime objective here is to enhance the ATC of the PS by indulging TCSC devices, which is shown in Eq. (3)

$$
\text { Obj= Improve (ATC) }
$$

During the incorporation of TCSC to ATC, the counts of the external devices connected by the users based on their requirements get reduced. Thus, in the clusters of transmission lines, the best connection denoted as $\left[C_{m}^{*}, D_{m}^{*}\right]$ is assessed. This is shown mathematically in Eq. (4), where $C_{m}$ and $D_{m}$ are said to be the indices of the clusters of lines of $\mathrm{m}^{\text {th }}$ TCSC and the "compensation level" of the same $\mathrm{m}^{\text {th }}$ TCSC bus system, respectively.

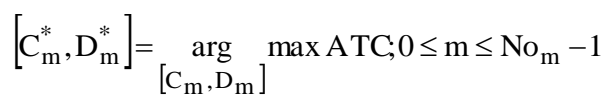

The main objective of introducing the GOAGA model is to select the branch in which the TCSC needs to be connected and the level of recompense that is to be fed into the bus system for achieving the improved ATC level. The solution encoding is given in Fig.3

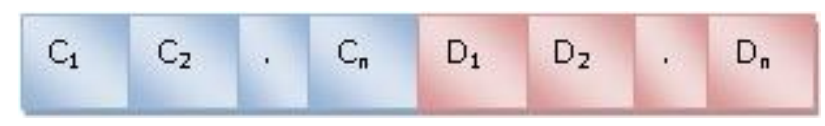

Fig. 3. Solution Encoding

\subsection{GOAGA Model}

The standardized GA is good in solving complex optimization problems, but it suffers from lower convergence. The convergence is faster in GOA and here the chance of getting trapped into local optimal is higher. Thus, both GA and GOA are linked together to form the GOAGA model. Steps followed in the proposed GOAGA model is explained below:

Step 1- Initialize the search agents of both GA and GOA. Let the current iteration be denoted as $t$. 
Step 2- Compute the fitness function for all the search agents.

Step 3- While $(\mathrm{t}<\max (\mathrm{t}))$, then proceed with the following steps or terminate the procedure.

Step 4- Then, using Eq. (5) update the value of a variable c , denoted as the lessening coefficient, with GOA model. Here, cmx refers to the utmost value, and cmn indicates the minimal value, $\mathrm{L}$ specifies the highest iteration count, and ${ }^{t}$ indicates the present iteration.

$$
\mathrm{c}=\mathrm{cmx}-\mathrm{t} \frac{\mathrm{cmx}-\mathrm{cmn}}{\mathrm{L}}
$$

Step 5- Then, normalize the distance between the grasshoppers between [1,4].

Step 6- Carry out the fitness computation as per Eq. (6) of the GA model.

$$
\mathrm{O}_{\mathrm{i}}=\frac{\sum_{\mathrm{i}=1}^{\mathrm{N}} \mathrm{D}_{\mathrm{ti}}}{\sum_{\mathrm{i}=1}^{\mathrm{N}} \mathrm{D}_{\mathrm{i}}}-\left[\frac{\sum_{\mathrm{i}=1}^{\mathrm{N}} \mathrm{D}_{\mathrm{i}}-\sum_{\mathrm{i}=1}^{\mathrm{N}} \mathrm{D}_{\mathrm{pi}}}{\sum_{\mathrm{i}=1}^{\mathrm{N}} \mathrm{D}_{\mathrm{i}}}\right]
$$

Consider $\mathrm{D}_{\mathrm{i}}, \mathrm{D}_{\mathrm{pi}}$ and $\mathrm{D}_{\mathrm{ti}}$ as bus line and $\mathrm{N}$ symbolizes the count of buses.

Step 7- On the basis of reduced fitness, the optimal two solutions are found.

Step 8- Update cross-over as per GA. The Crossover employs an application that relates to the genetic life of the parents.

Step 9- Carry out the fitness evaluation as per Eq. (5) of the GA model after the cross-over function.

Step 10- $\quad$ Merge both the best solutions attained from GOA and GA

Step 11- Discover the optimal solution from the merged results.

Step 12- The present search agent is brought back to the original position

Step 13- Update the value of $\mathrm{t}$ and increase it by $1(\mathrm{t}=\mathrm{t}+1)$. Then, check

Step 14- Then, move to step 3.

\section{Results and Discussion}

\subsection{Experimental Setup}

The ATC determination as well as enrichment with TCSC using GOAGA is constructed in MATLAB and tested on two IEEE test bus systems like IEEE 14 (with 3 GENCOs) and IEEE 39 (with 6 GENCOs). The evaluation is made in terms of statistical assessment. "In general, the meta-heuristic algorithms are stochastic and they aren't able to provide an accurate result. Hence, the statistical evaluation is conducted by executing the proposed as well as traditional model for five-time, and the corresponding performance measures like best, worst, mean, median, and standard deviation are determined". Further, the proposed ATC enhancement model is compared over the existing models like GA [23], PSO [17], MFO [18], and GOA [20].

\subsection{Statistical Evaluations on IEEE 14 Bus System: Varying the Count of TCSC}

The statistical evaluation is undergone by varying the count of TCSC connection. The resultant of the statistical evaluation for the "IEEE -14 bus system" is exhibited in Fig. 4. Here, the evaluation is done by varying the count of TCSCS buses as 1, 3, 5, and the corresponding results acquired are shown in Fig. 4(a), Fig. 4(b) and Fig. 4(c), respectively. Among all the statistical measures, the best performance as well as the mean performance acts as the key for verifying the presented work (GWOGA) over the extant assessments. In the case of best performance, the GWOGA model has exhibited the highest value of 135.98 , which is $8.4 \%, 3.6 \%, 27.6 \%$, and $28.7 \%$ much superior to the existing models like GA, PSO, MFO, and GOA, respectively. Then, the mean of the GWOGA is 146.93 , which is higher than GA= 124.26, $\mathrm{PSO}=119.7, \mathrm{MFO}=98.324$, and $\mathrm{GOA}=95.024$. Then, on observing Fig. 4(b), the connection of TCSC connected to the bus system is 3 . Here the best performance, worst performance, means performance, mean performance and standard deviation of the presented work are 143.85, 127.47, 145.32, 127.08, 10.5286, respectively. All these values are higher than the existing models. On observing the mean value, the GOAGA model is the highest one and it is $13.5 \%, 19.28 \%, 34.81 \%$, and $30.04 \%$ much superior to the existing models like GA, PSO, MFO, and GOA, respectively. Then, in Fig. 4(c), 5 counts of TCSC are connected to the bus system. Here, the highest best performance is recorded by the GOAGA model as 154.33 and it's the highest mean value is 144.3. Thus, from the evaluation, the GOAGA is good in enhancing the ATC for every variation in TCSC. 


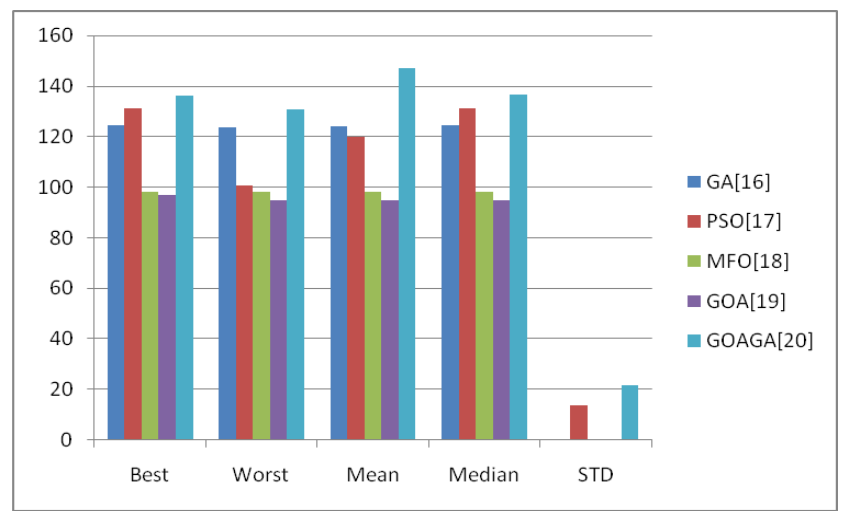

(a)

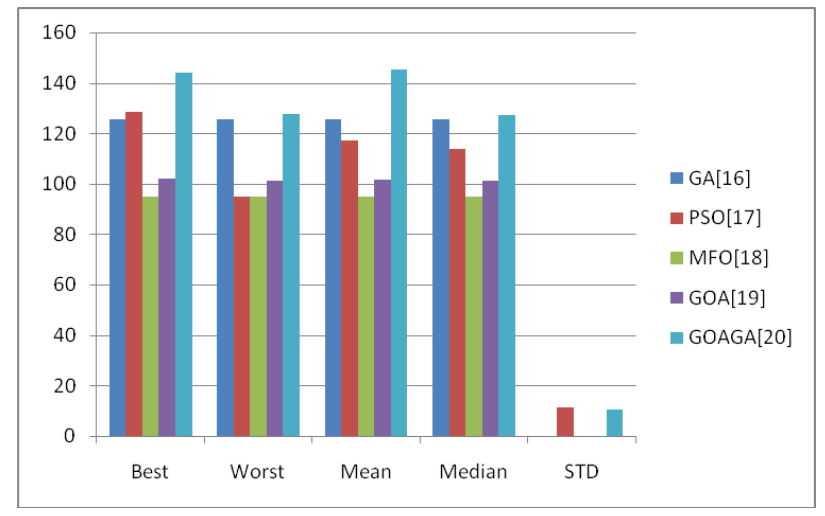

(b)

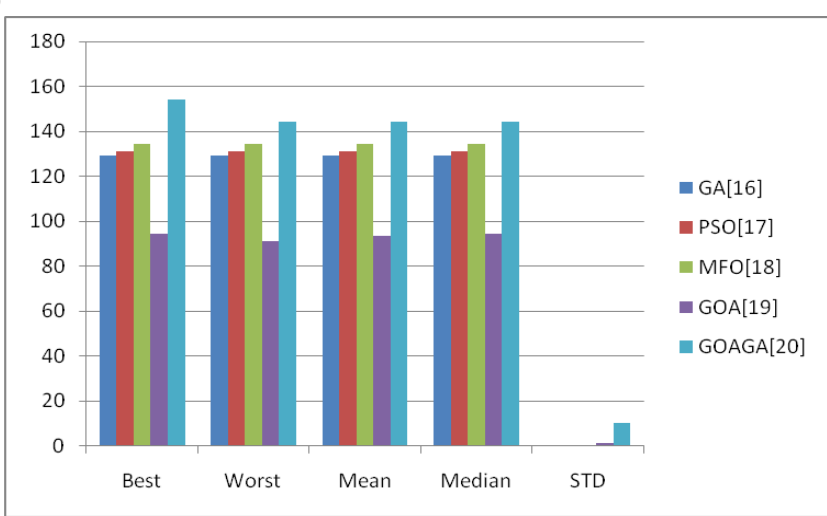

(c)

Fig. 4. Statistical evaluation of GOAGA over existing work under IEEE-14 bus system for variation in the count of TCSC (a) TCSC $=1$, (b) TCSC $=3$ and $T C S C=5$

\subsection{Statistical Evaluations on IEEE 39 Bus System: Varying the Count of TCSC}

Fig. 5 exhibits the statistical evaluation for the IEEE 39 bus system for a varying count of TCSCs. On observing, Fig. 5(a) with TCSC=1, the mean of GOAGA is $4.9 \%, 4.5 \%, 4.99 \%$, and $4.8 \%$ much superior to GA, PSO, MFO, and GOA, respectively. Then, in Fig. 4(b), the count of TCSC connected =3. Here, the best performance of GOAGA is the highest one and it is $4.6 \%, 11.92 \%, 11.92 \%$, and $11.89 \%$ much superior to GA, PSO, MFO, and GOA, respectively. The mean of GOAGA is 63.605, which is the highest one, while compared to $\mathrm{GA}=60.43, \mathrm{PSO}=60.69, \mathrm{MFO}=60.43$, and $\mathrm{GOA}=61.006$. Then, on observing, Fig. $5(\mathrm{c})$, the count of TCSC connected to the bus system is 5 . Here, the best performance of GOAGA is $7.2 \%$, $8.9 \%, 8.9 \%$, and 8.95 much superior to GA, PSO, MFO, and GOA, respectively. Then, the mean of GOAGA is 68.012 , which is $6.7 \%, 7.95,7.9 \%$, and $10.06 \%$, respectively. Thus, the GOAGA is said to be much adequate for ATC enhancement.

\section{Conclusion}

In this paper, a novel hybrid optimization method is introduced to enhance the ATC of the PS. Here, TCSC was incorporated as a tool for ATC enhancement. The novel algorithm referred as GOAGA was formulated by hybridizing the concept of GA s GOA. The GOAGA had aided in selecting the line in which TCSC needs to be connected and how much compensation need to be provided. Finally, the experimentation was done under two bench mark bus systems like IEEE 14 and IEEE 39. On observing, IEEE 39 bus system, the mean of GOAGA is 68.012 , which is $6.7 \%, 7.95,7.9 \%$ and $10.06 \%$, respectively. Thus, the GOAGA is said to be much adequate for ATC enhancement. 


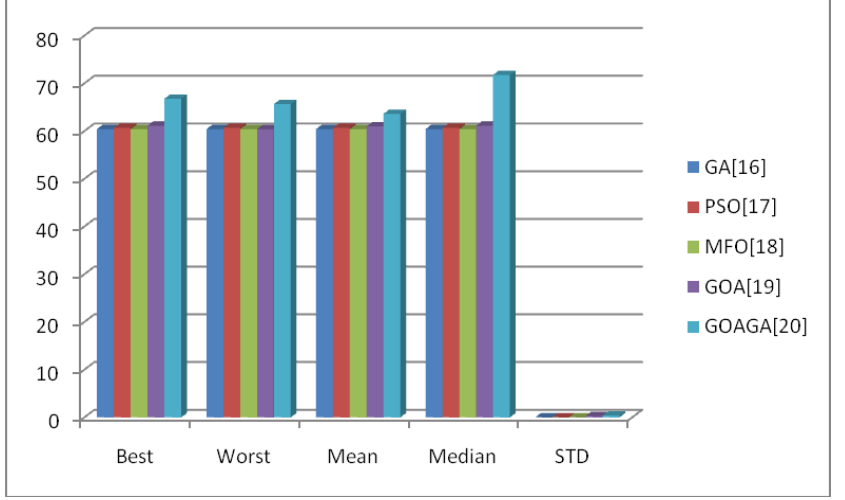

(a)

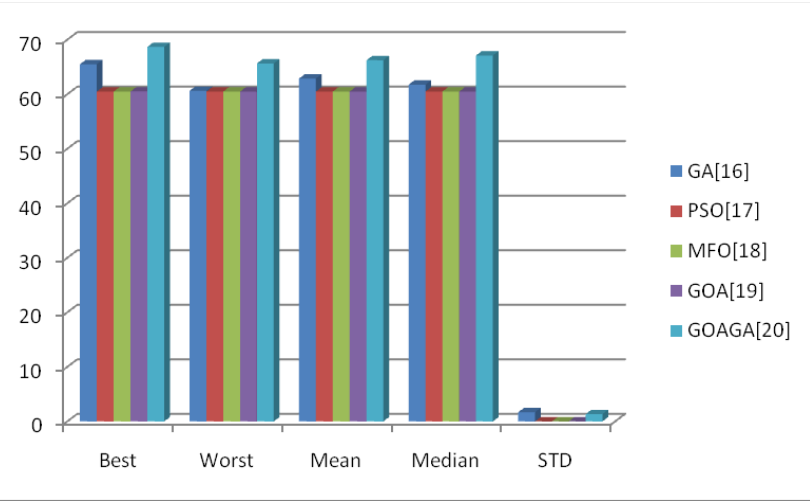

(b)

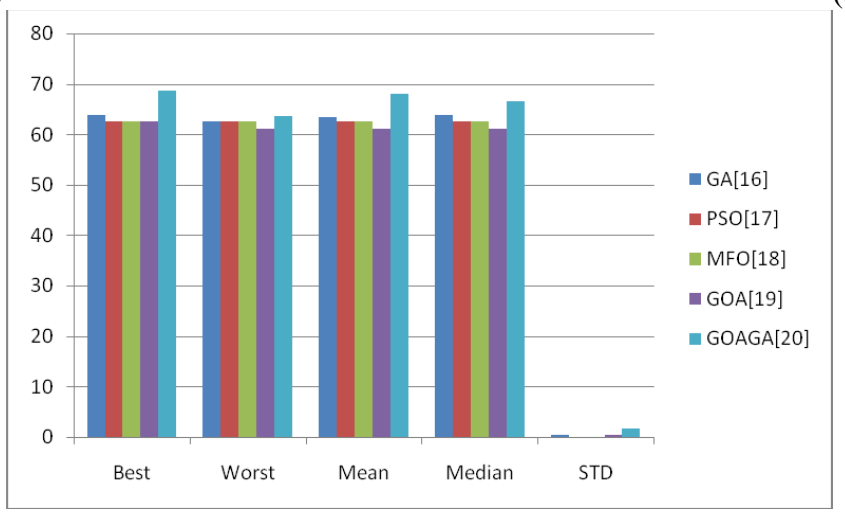

(c)

Fig. 5. Statistical evaluation of GOAGA over existing work under IEEE-39 bus sytem for varaition in the count of $\operatorname{TCSC}$ (a) $T C S C=1$, (b) $T C S C=3$ and $T C S C=5$

\section{References}

[1] P. Gopi KrishnaT. Gowri ManoharG. N. Srinivas,"Online Monitoring of Available Transfer Capability in Deregulated Power System Using Adaptive Neuro Fuzzy Inference System", Power Electronics and Instrumentation Engineering, pp 74-79,2010.

[2] A. Srinivasan, P. Venkatesh, B. Dineshkumar, N. Ramkumar,"Dynamic available transfer capability determination in power system restructuring environment using support vector regression",International Journal of Electrical Power \& Energy Systems,vol.69,pp.123-130, July 2015.

[3] M. Mohseni, A. Nouri, P. Salmanpor Bandaghiri, R. Saeedavi and M. Afkousi-Paqaleh, "Probabilistic Assessment of Available Transfer Capacity via Market Linearization," in IEEE Systems Journal, vol. 9, no. 4, pp. 1409-1418, Dec. 2015.

[4] T. Nireekshana, G. Kesava Rao, S. Sivanaga Raju,"Available transfer capability enhancement with FACTS using Cat Swarm Optimization",Ain Shams Engineering Journal,vol.7, no.1, pp. 159-167,March 2016.

[5] Gopi Krishna Pasam,T. Gowri Manohar,"Multi-objective Decision Based Available Transfer Capability in Deregulated Power System Using Heuristic Approaches",Journal of The Institution of Engineers (India): Series B, vol.97, no.3, pp. 385-393, September 2016.

[6] Ashwani Kumar, Jitendra Kumar,"ATC enhancement in electricity markets with GUPFC and IPFC - A comparison",International Journal of Electrical Power \& Energy Systems, vol.81,pp.469-482,2016

[7] Abhishek Gupta, Ashwani Kumar,"ATC Determination with Heuristic techniques and Comparison with Sensitivity based Methods and GAMS",Procedia Computer Science, vol.125,pp 389-397,2018

[8] Ahmad AL Ahmad, Reza Sirjani,"Optimal placement and sizing of multi-type FACTS devices in power systems using metaheuristic optimisation techniques: An updated review",Ain Shams Engineering Journal, in communication,2019

[9] Vladan Durković, Aleksandar S. Savić,"ATC enhancement using TCSC device regarding uncertainty of realization one of two simultaneous transactions",International Journal of Electrical Power \& Energy Systems, vol.115,February 2020

[10] Shashank Singh, Shiva Pujan Jaiswal,"Enhancement of ATC of micro grid by optimal placement of TCSC",Materials Today: Proceedings, in communication, 2020

[11] K. Bavithra, S. Charles Raja, P. Venkatesh,"Optimal Setting of FACTS Devices using Particle Swarm Optimization for ATC Enhancement in Deregulated Power System",IFAC-PapersOnLine, vol.49, no.1,pp.450455,2016

[12] M. V. k. Reddy and T. Nireekshana, "Enhancement of Available Transfer Capability using FACTS Devices," 2019 International Conference on Intelligent Computing and Control Systems (ICCS), Madurai, India, pp. 869874,2019 
[13] D. Shukla, S. P. Singh and S. R. Mohanty, "Optimal Strategy for ATC Enhancement and Assessment in presence of FACTS devices and Renewable Generation," 2018 20th National Power Systems Conference (NPSC), Tiruchirappalli, India, pp. 1-6,2018

[14] B. O. Adewolu and A. K. Saha, "Performance Evaluation of FACTS Placement Methods for Available Transfer Capability Enhancement in a Deregulated Power Networks," 2020 International SAUPEC/RobMech/PRASA Conference, Cape Town, South Africa, pp. 1-6,2020

[15] Sidnei do Nascimento, Maury M. Gouvêa,"Voltage Stability Enhancement in Power Systems with Automatic Facts Device Allocation",Energy Procedia, vol.107,pp.60-37,2017

[16] JohnMcCall, " Genetic algorithms for modelling and optimisation", Journal of Computational and Applied Mathematics, vol. 184, no. 1, pp. 205-222, 2005.

[17] M.R.Tanweer, S.Suresh, and N.Sundararajan, " Self regulating particle swarm optimization algorithm", Information Sciences, vol. 294, pp. 182-202, 2015.

[18] Seyedali Mirjalili,"Moth-flame optimization algorithm: A novel nature-inspired heuristic paradigm",KnowledgeBased Systems,vol.89, pp.228-249,November 2015

[19] ShahrzadSaremi,SeyedaliMirjalili,AndrewLewis,"GrasshopperＯ Optimisation～Algorithm: Theory and application",Advances in Engineering Software, Vol.105, pp.30-47, 2017

[20] M.M.Annie Alphonsa and N.MohanaSundaram,"A reformed grasshopper optimization with genetic principle for securing medical data",Journal of Information Security and Applications, vol.47,pp.410-420, 2019

[21] Rajakumar B R, "Optimization using lion algorithm: a biological inspiration from lion's social behavior", Evolutionary Intelligence, Special Issue on Nature inspired algorithms for high performance computing in computer vision, Vol. 11, No. 1-2, pages 31-52, 2018, DOI: https://doi.org/10.1007/s12065-018-0168-y.

[22] B. R. Rajakumar, "Impact of Static and Adaptive Mutation Techniques on Genetic Algorithm", International Journal of Hybrid Intelligent Systems, Vol. 10, No. 1, pages: 11-22, 2013, DOI: 10.3233/HIS-120161

[23] B. R. Rajakumar, "Static and Adaptive Mutation Techniques for Genetic algorithm: A Systematic Comparative Analysis", International Journal of Computational Science and Engineering, Vol. 8, No. 2, pages: 180-193, 2013, DOI: 10.1504/IJCSE.2013.053087

[24] B. R. Rajakumar, "Lion algorithm for standard and large scale bilinear system identification: A global optimization based on Lion's social behavior", 2014 IEEE Congress on Evolutionary Computation, Beijing, China, July 2014, pages: 2116-2123, DOI: 10.1109/CEC.2014.6900561

[25] S. M. Swamy, B. R. Rajakumar and I. R. Valarmathi, "Design of Hybrid Wind and Photovoltaic Power System using Opposition-based Genetic Algorithm with Cauchy Mutation", IET Chennai Fourth International Conference on Sustainable Energy and Intelligent Systems (SEISCON 2013), Chennai, India, Dec. 2013, DOI: 10.1049/ic.2013.0361 